\title{
Caspase cleavage of viral proteins, another way for viruses to make the best of apoptosis
}

\author{
A Richard ${ }^{\star, 1}$ and D Tulasne ${ }^{1}$
}

Viral infection constitutes an unwanted intrusion that needs to be eradicated by host cells. On one hand, one of the first protective barriers set up to prevent viral replication, spread or persistence involves the induction of apoptotic cell death that aims to limit the availability of the cellular components for viral amplification. On the other hand, while they completely depend on the host molecular machinery, viruses also need to evade the cellular responses that are meant to destroy them. The existence of numerous antiapoptotic products within the viral kingdom proves that apoptosis constitutes a major threat that should better be bypassed. Among the different strategies developed to deal with apoptosis, one is based on what viruses do best: backfiring the cell on itself. Several unrelated viruses have been described to take advantage of apoptosis induction by expressing proteins targeted by caspases, the key effectors of apoptotic cell death. Caspase cleavage of these proteins results in various consequences, from logical apoptosis inhibition to more surprising enhancement or attenuation of viral replication. The present review aims at discussing the characterization and relevance of this post-translational modification that adds a new complexity in the already intricate host-apoptosis-virus triangle.

Cell Death and Disease (2012) 3, e277; doi:10.1038/cddis.2012.18; published online 8 March 2012

Subject Category: Immunity

\section{Apoptosis Faces Up Viruses: Die Hard}

When a viral infection threatens cells, one of the first measures they take is to induce apoptosis to restrict viral replication and spread. ${ }^{1-3}$ Dying this way, host cells are likely to generate specific signals aiming at triggering the immune system with innate and/or adaptative responses allowing the eradication of the invader. ${ }^{4,5}$ On the other hand, viruses have evolved a huge arsenal of strategies meant to either counteract or deal with this destructive process to ensure their survival. ${ }^{6-8}$ Apoptotic cell death is accompanied by characteristic morphological changes (cellular rounding-up and volume reduction, chromatin condensation, nuclear fragmentation, plasma membrane blebbing...) and at a molecular level by the activation of first initiator and then effector cysteinyl aspartate proteinases or caspases. ${ }^{9,10}$ Activated caspases act through a catalytic Cys that hydrolyzes peptide bonds within the substrate, with a stringent specificity for Asp residue at $\mathrm{P} 1$ position (the nature of residues at positions $\mathrm{P} 2$, P3 and P4 depending on the caspase). Caspase substrates include a large number and variety of cellular proteins that participate through their cleavage to the strong apoptosisrelated morphological changes, as well as other physiological processes. Interestingly, viral proteins are also likely to be cleaved by caspases but until 2004, only four of them were reported as such and suggested as advantaging the associated viruses through their cleavage. ${ }^{11}$ Since, their number has greatly increased and at least 16 viruses are now known to express proteins that undergo caspase cleavage. Here, we aim at updating these cleavages and discussing their biological relevance. From apoptosis inhibition to the improvement or attenuation of viral amplification, caspase cleavage of VPs highlights a potential new fascinating viral strategy to handle apoptosis induction.

\section{Caspase Cleavage as a Way for Viruses to Counteract Apoptosis: Die Another Day}

Apoptosis induction by infected cells is meant to jeopardize viral replication. Therefore, many viruses have developed strategies to inhibit this process through various mechanisms. Among them, inhibiting caspase activity through the cleavage of viral proteins constitutes an effective way to achieve apoptosis suppression (Table 1).

Baculoviruses are invertebrate viruses that encode several antiapoptotic products including IAPs, P49 and P35. When lacking P35, the baculovirus prototype Autographa californica multicapsid nucleopolyhedrovirus (AcMNPV) induces apoptosis and fails to replicate, whereas P35 rescue inhibits cell death and restores viral replication. ${ }^{12}$ Interestingly, P35 is proteolytically cleaved on infection ${ }^{13,14}$ and the ${ }^{84} \mathrm{DQMD}^{87} \downarrow$ cleavage site is required for P35-mediated apoptosis suppression. P35 directly inhibits many caspases, including

${ }^{1}$ Centre National de la Recherche Scientifique (CNRS) Unité mixte de recherche (UMR) 8161, Institut de Biologie de Lille, Institut Pasteur de Lille, Université Lille-Nord de France, Institut fédératif de recherche (IFR) 142, Lille, France

${ }^{*}$ Corresponding author: A Richard, Centre National de la Recherche Scientifique (CNRS) Unité mixte de recherche (UMR) 8161, Institut de Biologie de Lille, Institut Pasteur de Lille, Université Lille-Nord de France, Institut fédératif de recherche (IFR) 142, Lille, France. Tel: + 33320871257 ; Fax: + 33320871111 ; E-mail: audreyrichard10@gmail.com

Keywords: virus; apoptosis; caspase cleavage; viral subversion

Abbreviations: caspase, cysteinyl aspartate proteinase; WT, wild-type; NS, non-structural protein; VP, viral protein

Received 07.12.11; revised 05.1.12; accepted 09.1.12; Edited by HU Simon 
insect Sf-caspase 1, human caspases 1, 3, 6, 7, 8 and 10 and mouse caspase $1 .{ }^{14,15}$ Indeed, P35 cleavage products remain irreversibly associated to the caspase through a covalent thioester bond between P35 D87 residue and the caspase catalytic cysteine. ${ }^{16,17}$ Although being $50 \%$-related to P35, P49 yet exhibits a different caspase cleavage site $\left({ }^{91}\right.$ TVTD $\left.^{94} \downarrow\right)$ that confers to P49 its antiapoptotic properties. ${ }^{18}$ Caspase suppression by P49 also involves its cleavage by and stable association to the targeted caspases although acting as a dimer. ${ }^{19}$ Besides effector caspases, P49 also affects initiator caspases that P35 fails to suppress, like insect Sf-caspase $X$ and human initiator caspase 9 .

Rather similarly, the product of the orf390 gene expressed by the crustacean-infecting white spot syndrome virus (WSSV) was also shown to exert antiapoptotic properties. Insect cells SF9 stably expressing orf390 gene strongly resist both viral- and actinomycin D-induced apoptosis. ${ }^{20}$ Like P35 and P49, ORF390 (also referred as WSSV449 or AAP-1) is suggested to act as an inhibitor substrate ${ }^{21}$ and is able to block several caspases, including human caspases 3 and 9 as well as insect Sf-caspase 1 in vitro. ORF390 ability to suppress caspase activity is associated with consensual caspase cleavage sites: ${ }^{269} \mathrm{DEVD}^{272} \downarrow$ that targets caspase 3 , and ${ }^{230} V_{E T D}{ }^{233} \downarrow$ and ${ }^{300}$ LEHD $^{303} \downarrow$ that are both required for caspase 9 inhibition. Although each site specifically targets one protease, the three of them are needed to reach maximal caspase inactivation. ${ }^{20}$

For baculoviruses and WSSV, inhibiting apoptosis is crucial to achieve their life cycle. These different invertebrateinfecting viruses encode proteins exhibiting active caspase cleavage sites, just as cellular substrates do, but with the special ability to irreversibly freeze the protease activity. This leads to a very potent and broad caspase inhibition, which keeps the host cell machinery available and fit for viral amplification.

\section{Caspase Cleavage as a Way for Viruses to Replicate and Spread: Live and Let Die}

Unlike the expression of antiapoptotic products, the requirement of apoptosis for viruses to survive is a way more counterintuitive strategy that yet has been evolved by several viruses and involves caspase cleavage (Table 2).

Parvovirus Aleutian mink disease virus (AMDV) can lead, at a single cell level, to either permissive infection, namely high levels of both viral DNA replication and production of viruses, or persistent infection with low viral DNA replication and almost no production of progeny virions. On permissive infection, AMDV induces caspase activation that is necessary for viral amplification. ${ }^{22}$ This requirement was associated with NS1 protein being cleaved through two caspase 3 sites leading to five NS1-related products. ${ }^{23}$ When one site is mutated within AMDV molecular clones, the viral production is strongly reduced and even aborted when both sites are disrupted. Interestingly, wild-type (WT) NS1 protein, which exerts replicative and transcriptional functions, is mostly nuclear, but when one of its cleavage sites is disrupted, the protein remains cytosolic resulting in a dramatic decrease in NS1-dependent viral protein (VP) expression. NS1-related C-terminal products, also nuclear, are suggested to be actively 


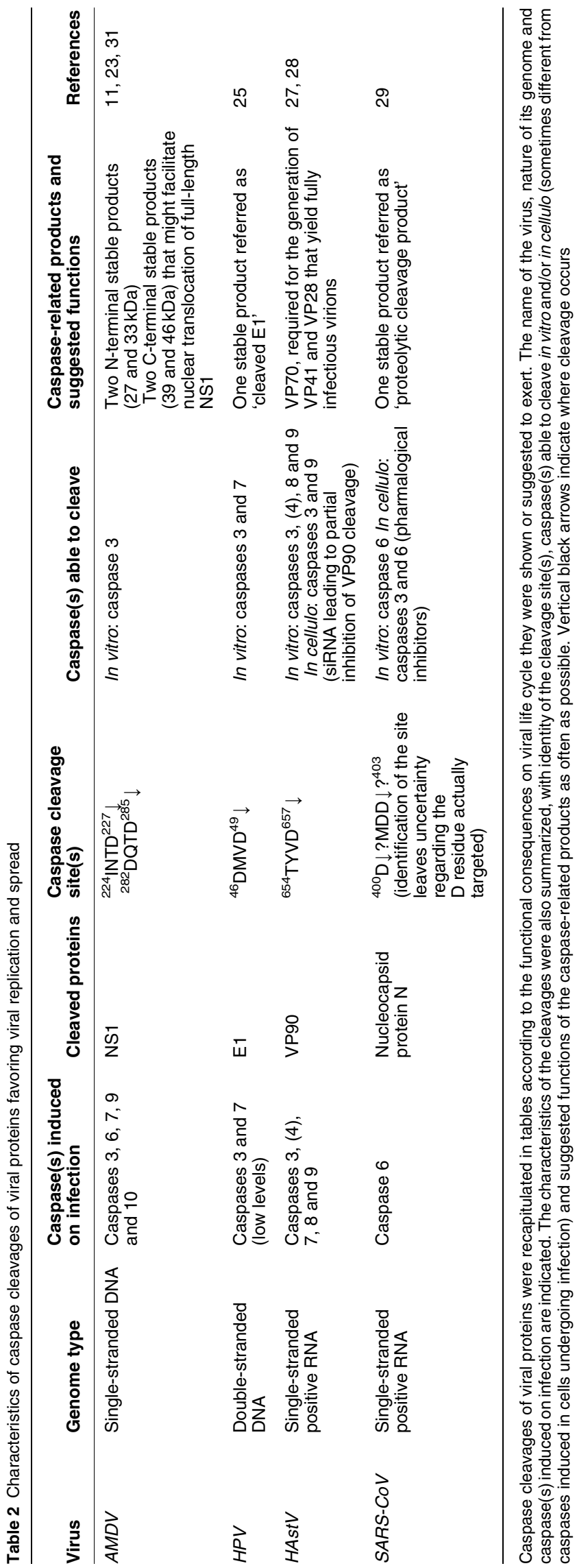

involved in the transport of WT NS1. Therefore, on apoptosis induction, full replication occurs, leading to permissive infection. Conversely, without caspase activation, AMDV amplification would be limited and that might allow this lytic virus to establish persistent infection.

Human papillomavirus (HPV) is mostly known to infect epithelial cells of the genital tract and cause cervical cancers. On epithelial differentiation, HPV induces a DNA damage response that leads to caspase 3-dependent apoptosis. ${ }^{24}$ Interestingly, HPV E1, a protein involved in viral DNA replication, is a target for caspases 3 and 7 at a site that is conserved in all genital HPVs and preventing E1 cleavage reduces viral amplification. When compared with chemically induced apoptosis, HPV-induced apoptotic markers are limited. Interestingly, HPV increases the levels of both antiapoptotic Bcl2 and Survivin proteins. ${ }^{25} \mathrm{HPV}$ pro- and antiapoptotic properties might achieve a caspase activity threshold that is sufficient for $E 1$ cleavage and viral amplification, but not lethal for the host cell. Besides E1, HPV E6 protein is cleaved by caspases as well, but the functional consequences remain unknown. ${ }^{26}$

Human astroviruses (HAstV) are responsible for gastroenteritis. In Yuc8 strain, orf2 encodes the precursor of viral capsid proteins, VP90, whose processing yields VP70. Besides, HAstV activates caspases $3,4,6,7,8$ and 9. ${ }^{27,28}$ In vitro, caspases 3, 8 and 9 are able to target $\mathrm{VP}^{27}$ but only caspase 3 or 9 silencing reduces VP70 generation on infection. Interestingly, both intracellular VP90 processing into VP70 and progeny virion release are lost in the presence of a pan caspase inhibitor. However, in opposition to what was first suggested, viral release needs caspase activation but does not need VP90 processing into VP70 to be achieved. ${ }^{27}$ But yielding fully infectious viruses does require shorter polypeptides to be created through VP70 further processing by trypsin. Thus, first cleaving VP90 to generate VP70 likely represents a crucial intermediate event during HAstV infection.

SARS-coronavirus (SARS-CoV) is a fairly new RNA virus responsible for a severe acute respiratory disease. SARSCoV leads to either permissive or persistent infection, with strong cytopathic effects and high viral titers or the opposite, respectively. Interestingly, the nucleocapsid protein $(N)$, that has both structural and non-structural functions, is proteolytically processed in cells undergoing a lytic cycle or ectopically expressing the protein. ${ }^{29}$ Using a pharmalogical approach, $\mathrm{N}$ protein was demonstrated to be targeted by caspases 3 and 6 . On permissive infection, $\mathrm{N}$ protein localizes in both cytosol and nucleus while remaining cytosolic in cells undergoing persistent infection. Moreover, preventing $\mathrm{N}$ from translocating to the nucleus by mutating its nuclear localization signal abolishes its cleavage by caspases. Thus, permissive infection is associated with $\mathrm{N}$ being translocated to the nucleus and possibly its ensuing caspase cleavage. Nevertheless, the mechanisms underlying viral replication, $\mathrm{N}$ subcellular localization and $\mathrm{N}$ caspase processing are unknown so far.

In sharp contrast with baculoviruses and WSSV, several very different viruses evolved to hijack apoptosis with caspase cleavage being required to reach optimal viral amplification. Based on the well-described case of AMDV, we can 
hypothesize that the caspase-related products created through cleavages display novel properties required for the achievement of the viral life cycle.

\section{Caspase Cleavage as a Way for Viruses to Downregulate Their Own Expression: You Only Live Twice}

In some cases, apoptosis induction by the host cell leads to exactly what is expected, namely viral attenuation, but surprisingly with the help of viral protein cleavages. The possible viral advantage resulting from such an event is discussed (Table 3).

Kaposi sarcoma-associated herpesvirus (KSHV) establishes long-term infections leading to Kaposi sarcomas and can go through either latent or lytic cycle. When KSHV is reactivated, ORF57 (or Mta or KS-SM) is detected by western blot as a doublet, ${ }^{30}$ with the smaller product being abolished by a pan caspase inhibitor. In vitro, caspase 7 , which is activated on KSHV reactivation, and to a lesser extent caspases 5 and 10 , are able to process ORF57 at ${ }^{30}$ DETD ${ }^{33} \downarrow$. Interestingly, coexpression assays show that ORF57 cleavage product (i.e. ORF57 lacking residues $1-33$ ) is no longer able to promote viral lytic gene expression. Accordingly, complementing a stable cell line containing an orf57-null KSHV genome with ectopic WT or uncleavable ORF57 promotes lytic gene expression, while ORF57 lacking residues 1-33 does not. Consistently, the number of cell-free virus particles dramatically increases when caspase 7 is inhibited. Thus, ORF57 cleavage would prevent proper expression of its downstream targets and subsequent full reactivation of $\mathrm{KSHV}$, suggesting that it allows $\mathrm{KSHV}$ to maintain a persistent infection.

As described above, AMDV NS1 protein is cleaved by caspases, which allows full viral replication. Interestingly, AMDV capsid proteins VP1 and 2 are also processed by caspases on viral infection or ectopic expression. ${ }^{31}$ The cleavage generates a stable $26-\mathrm{kDa}$ product, VPx, and can be partly prevented by the pharmacological inhibition of caspases $6,8,9$ and 10, and completely by a pan caspase inhibitor. Besides, VP protein expression in either transfected or AMDV-infected cells mainly activates caspase 10 , followed by caspases $6,9,3$ and 7 , with caspase 7 as the more efficient in VP cleavage in vitro. Altogether, these data argue for the involvement of several caspases in VP cleavage. AMDV-G strain grows more efficiently at $31.8^{\circ} \mathrm{C}$ than $37^{\circ} \mathrm{C}$. The alteration of the caspase site within AMDV-G increases both VP and genomic copies production at the non-optimal temperature of $37^{\circ} \mathrm{C}$ and shows no significant effects at $31.8^{\circ} \mathrm{C}$, suggesting that VP caspase cleavage attenuates AMDV-G life cycle. In vivo AMDV-G infection might thus be regulated by active caspases because it only occurs at $37^{\circ} \mathrm{C}$. Interestingly, NS1 cleavage favors viral DNA replication while VP processing would limit the packaging of progeny virions. As it is possible that both NS1 and VP cleavages do not inevitably occur simultaneously depending on the pattern of activated caspases, it would result in either effective or attenuated viral replication and allow the virus to spread or persist.

H-1 parvovirus (H-1PV) displays oncotropic and oncolytic features meaning it preferentially replicates in and kills

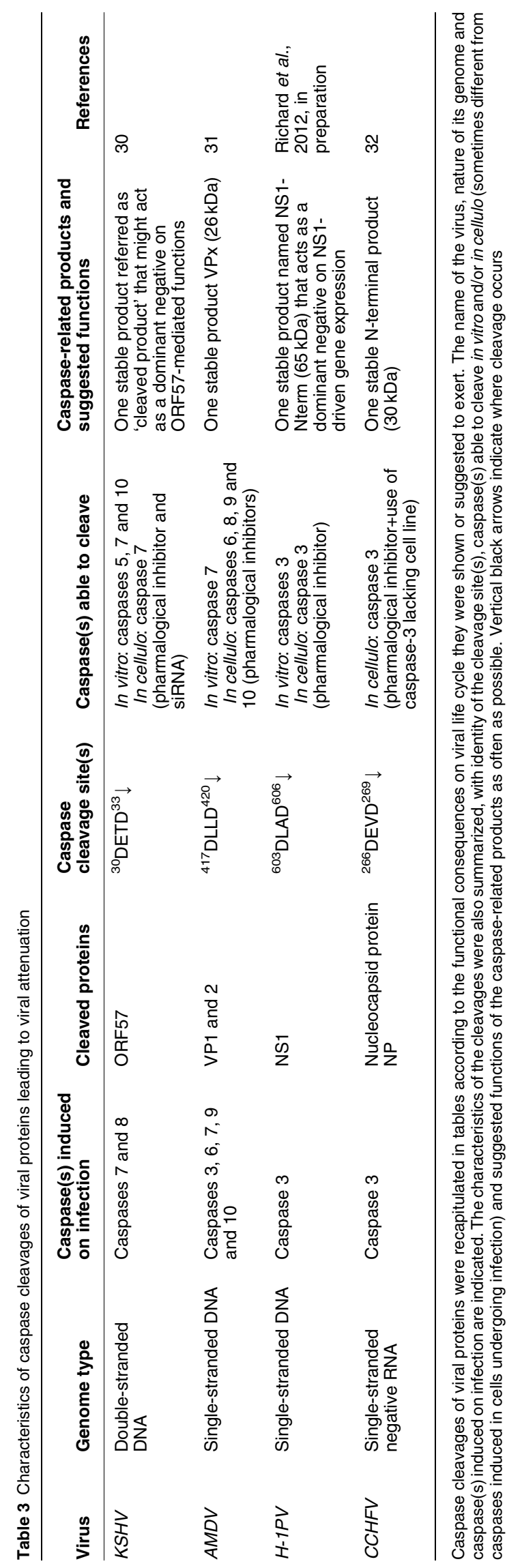


transformed cells, mainly in a non-apoptotic manner. However $\mathrm{H}-1 \mathrm{PV}$ is able to activate caspases in non-transformed cells, leading to the cleavage of NS1, a non-structural protein (NS) notably involved in viral DNA replication and gene expression by transactivating P38 promoter, which controls the synthesis of capsid proteins. Cleaved NS1 protein (named NS1-Nterm) lacks its C-terminal transactivation domain. When ectopically expressed, NS1-Nterm acts as a dominant negative on NS1-driven P38 promoter transactivation, and dramatically decreases viral production. Moreover, a molecular clone expressing an uncleavable version of NS1 tends to generate more virions. As cancer cells are often refractory to proper apoptotic induction, we propose that $\mathrm{H}-1 \mathrm{PV}$ oncotropism is due, at least in part, to the inability of cancer cells to cleave NS1 protein, thus allowing strong virus production. On the other hand, NS1 cleavage would act as a sensor of antiviral defenses and accordingly attenuate viral amplification, likely to favor persistent infection (our unpublished results).

Crimean-Congo hemorrhagic fever virus (CCHFV) causes severe coagulation dysfunction in humans. CCHFV nucleocapsid protein (NP) is an important structural protein also involved in viral replication and, when ectopically expressed with an apoptotic factor such as Bax protein, it generates two fragments through cleavage at a consensual ${ }^{266} \mathrm{DEVD}^{269} \downarrow$ motif. ${ }^{32}$ A caspase 3 inhibitor and cells lacking caspase 3 both allows a one log-increase in the production of virions, which suggests that caspase activation is involved in CCHFV attenuation. Thus, NP caspase cleavage and/or NP-related products may participate to this attenuation although the mechanisms remain to be investigated. Interestingly, the caspase cleavage site is conserved within all the strains that were checked, suggesting that it is an important feature for CCHFV regulation.

Caspase cleavages of viral proteins can result in the attenuation of the virus. Rather than a weakness of the virus, this might be considered as a way for the virus to adapt to the cellular context: as a result of such viral attenuation, the host response is largely reduced while the virus keeps its ability to replicate to some extent.

\section{Unknown Functions of viral protein Caspase Cleavages: On her Majesty's Secret Service}

Although the consequences of several caspase protein cleavages have been elucidated, they remain elusive so far for others (Table 4).

Influenza viruses are structured into ribonucleoprotein segments consisting of viral RNA and viral proteins, the major one being the NP. Unlike avian NP, NP proteins from human influenza $A$ and $B$ viruses are long known to be caspase targets. ${ }^{33,34}$ Human influenza with uncleavable ('avian-like') NP is dramatically less lethal for mice, along with lower viral titers and faster clearance than its WT counterpart. This argues for the involvement of NP caspase cleavage in the virulence of influenza virus. But making avian NP cleavable (i.e. making the protein 'human-like' by introducing the same caspase cleavage site) does not enhance the viral virulence as could have been expected. Thus, modulating influenza pathogenicity through NP caspase cleavage would be a specificity of human strains that cannot be extrapolated to avian ones. ${ }^{35}$ However, avian influenza pathogenicity might still be regulated by caspase cleavage. The viral ionic channel M2 protein is also cleaved by caspases, likely caspase 2 and/ or $3^{36,37}$ in both human and avian influenza viruses and was shown to be associated with avian influenza pathogenicity. ${ }^{37}$ Altogether, these studies highlight that modulating influenza caspase sites attenuates the virus although through currently unknown mechanisms. ${ }^{37,38}$

Hepatitis $C$ virus (HCV) often leads to chronic infection evolving to cirrhosis and possibly hepatocellular carcinoma. $\mathrm{HCV}$ core protein induces activation of caspases 2 and 6 , and interacts with viral NS5A protein that is cleaved by the activated caspases. ${ }^{39-42}$ Several studies point to a role of NS5A cleavage in its subcellular localization. The protein is mainly found in the cytosol while exerting nuclear functions. NS5A caspase cleavage was first suggested to allow the removal of a C-terminal cytoplasmic retention signal along with the translocation of the C-terminal deleted NS5A to the nucleus, reminding what was already described for AMDV NS1 protein. ${ }^{42}$ However, a study more recently reported a cytosolic localization of these C-terminal truncated forms of NS5A and no obvious role of NS5A caspase cleavage in its trafficking. ${ }^{40}$ Although its characterization is well admitted, the functional relevance of NS5A cleavage is still debated.

Transmissible gastroenteritis coronavirus (TGEV) causes acute and fatal diarrhea in newborn piglets. TGEV infection is able to induce caspases $3,6,7,8$ and 9 activation ${ }^{43,44}$ and the cleavage of the structural NP N, leading to a stable $41-\mathrm{kDa}$ fragment $\left(\mathrm{N}^{\prime}\right) .{ }^{44} \mathrm{~N}$ protein is targeted in vitro by caspases 6 and 7 , and less efficiently by caspase 3 . In HRT18jap1 cells, the infection causes apoptosis but is not productive, suggesting that caspase activation (and possibly $\mathrm{N}$ caspase cleavage) prevents progeny virion generation. However, caspase inhibition in this context does not restore any viral production. Owing to the lack of satisfactory tools, human HRT18jap1 cells were used as a study model but might not be appropriate to reveal $\mathrm{N}$ caspase cleavage relevance, notably for virus production. Further digging will thus be needed to understand the biological significance of $\mathrm{N}$ processing by caspases on TGEV infection.

Feline calicivirus (FCV) causes upper respiratory tract disease in cats. FCV capsid protein is synthesized as a precursor further cleaved by the viral protease. However, a $40-\mathrm{kDa}$ product (P40) is also usually observed at late stages of FCV infection and can be abolished with a pan caspase or a caspase $3 / 7$ inhibitor. $^{45}$ Accordingly, FCV-infected cells undergo apoptosis as well as caspases 2, 3 and 7 activation. ${ }^{46}$ Only caspase 2 and far less efficiently caspase 6 process FCV capsid protein in vitro at cleavage sites that have not been described yet. As suggested for instance for AMDV, cleavage of the capsid protein might impair particle assembly and participate to viral persistence but this has not been investigated either.

Adenoviruses gather more than a 100 species with about 40 being able to infect humans. Adenovirus early region $1 \mathrm{~A}$ (AdE1A) encodes two major proteins, $12 \mathrm{~S}$ and 13S, that are involved in the control of early viral gene expression through their interactions with the host cell machinery. In vitro, caspases 3 and 7 are able to cleave both 12S and 13S proteins, although the cleavage efficiency depends on the 


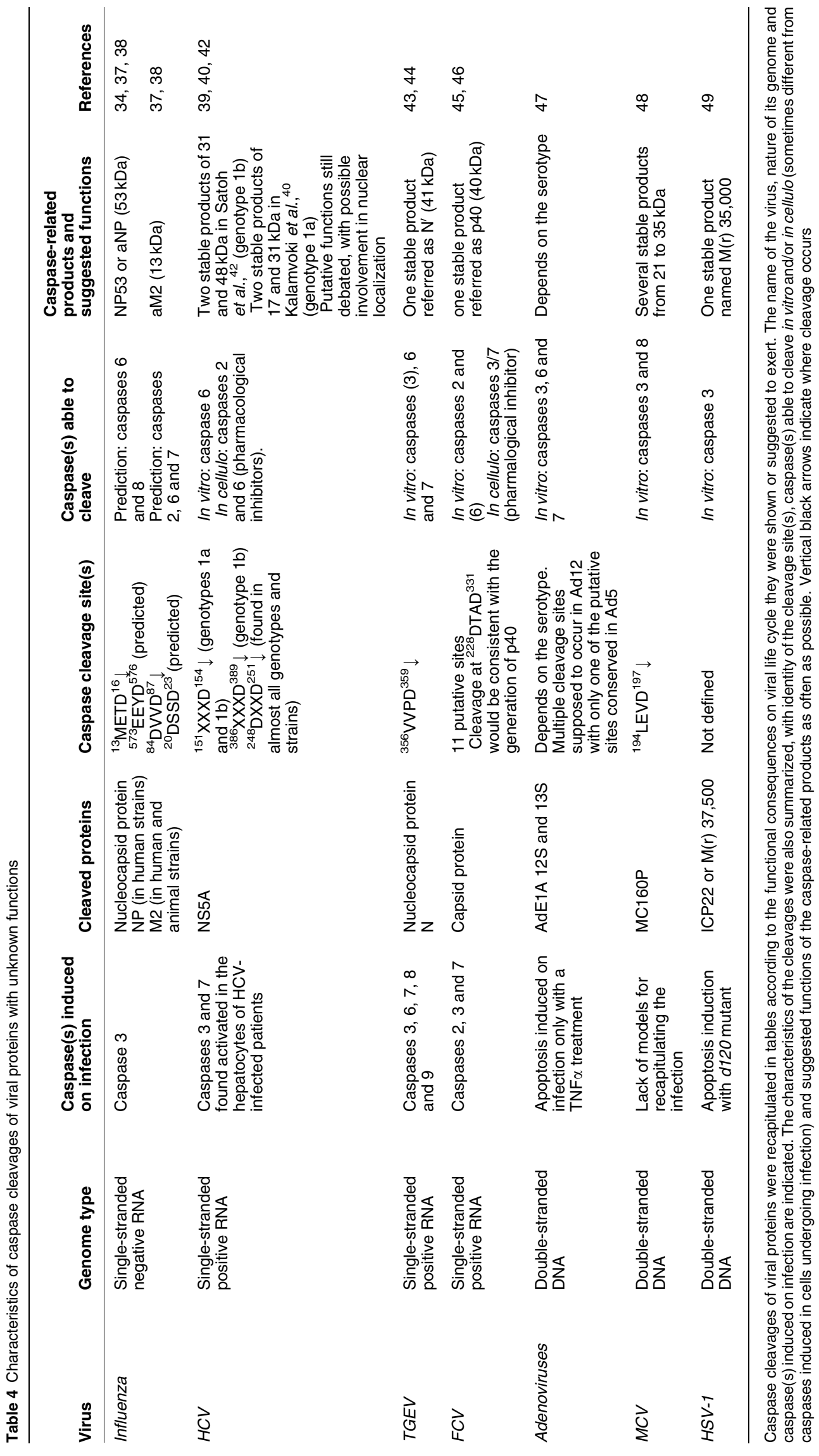


viral serotype. Furthermore, when apoptosis is chemically induced in AdE1A-transformed cells, AdE1A levels decrease but can be restored using a pan caspase inhibitor. ${ }^{47}$ However, AdE1A levels remain stable on adenovirus infection despite TNF $\alpha$-induced caspase activation. Thus, although AdE1A caspase cleavage is likely to occur, its existence on infection as well as its biological relevance for adenoviruses remains elusive so far. Interestingly, AdE1A-binding abilities to transcription factors such as CBP and TBP are affected by the cleavage, suggesting that it might modulate the expression of early viral genes.

Molluscum contagiosum virus (MCV) is a poxvirus that, like variola, exclusively infects humans. MC159P, an MCV protein, exhibits antiapoptotic properties by protecting cells from Fas-induced apoptosis through caspase 8 inhibition but is not targeted by caspases. ${ }^{48}$ On the other hand, MC160P is not associated with any antiapoptotic abilities but in vitro, is cleaved by caspases 3 and $8 .{ }^{48}$ When expressed through vaccinia virus, MC160P is also specifically cleaved by caspases on Fas-induced apoptosis while MC159P remains stable, as expected. MC159P and MC160P being thought to be produced simultaneously, a balance might take place between MC159P antiapoptotic response and MC160P caspase cleavage. However, the pathophysiological significance of this balance is difficult to address because MCV does not grow in tissue culture or experimental animals.

Herpex simplex virus 1 (HSV-1) causes lifelong infections affecting between 50 and $90 \%$ of the global population. HSV-1 exerts antiapoptotic properties against numerous stimuli. But some variants including d120 mutant induce apoptosis and under these conditions, infected cell protein no. 22 (ICP22 or $M_{r} 37,500$ protein) leads to an additional product $\left(M_{r} 35,000\right)$ that can result in vitro from ICP22 cleavage by caspase $3{ }^{49}$ Neither the identity of the caspase site nor the biological relevance of ICP22 cleavage has been further investigated yet.

Viruses Face Up to Apoptosis: Die Harder. There are clear evidences concerning the relevance of viral protein caspase cleavages in the physiology of viruses (Figure 1). Coherently, such a modification is used to directly fight apoptosis and AcMNPV as well as WSSV express proteins acting as strong and broad inhibitor caspase substrates. For other viruses, caspase cleavage probably allows the removal of specific regions that reveals or eliminates functional domains or signals. In some cases, this leads to full viral amplification (AMDV NS1, HPV E1), with the striking example of AMDV NS1 protein that needs to be cleaved by caspases to translocate to the nucleus and eventually exert its functions. On the contrary, caspase cleavage can result in the attenuation of the virus by generating new viral products acting as dominant negatives of NS proteins ( $\mathrm{H}-1 \mathrm{PV}$ NS1, KSHV ORF57) or unfit for virus packaging when structural proteins are concerned (AMDV VP and possibly FCV capsid protein). Interestingly, such attenuation at a cellular level might hold importance regarding the possibility for viruses to establish permissive and/or persistent infection at a higher scale (i.e. multicellular organisms). The functional consequences of some viral protein caspase cleavages still remain debated or elusive because of the difficulty of investigating hazardous

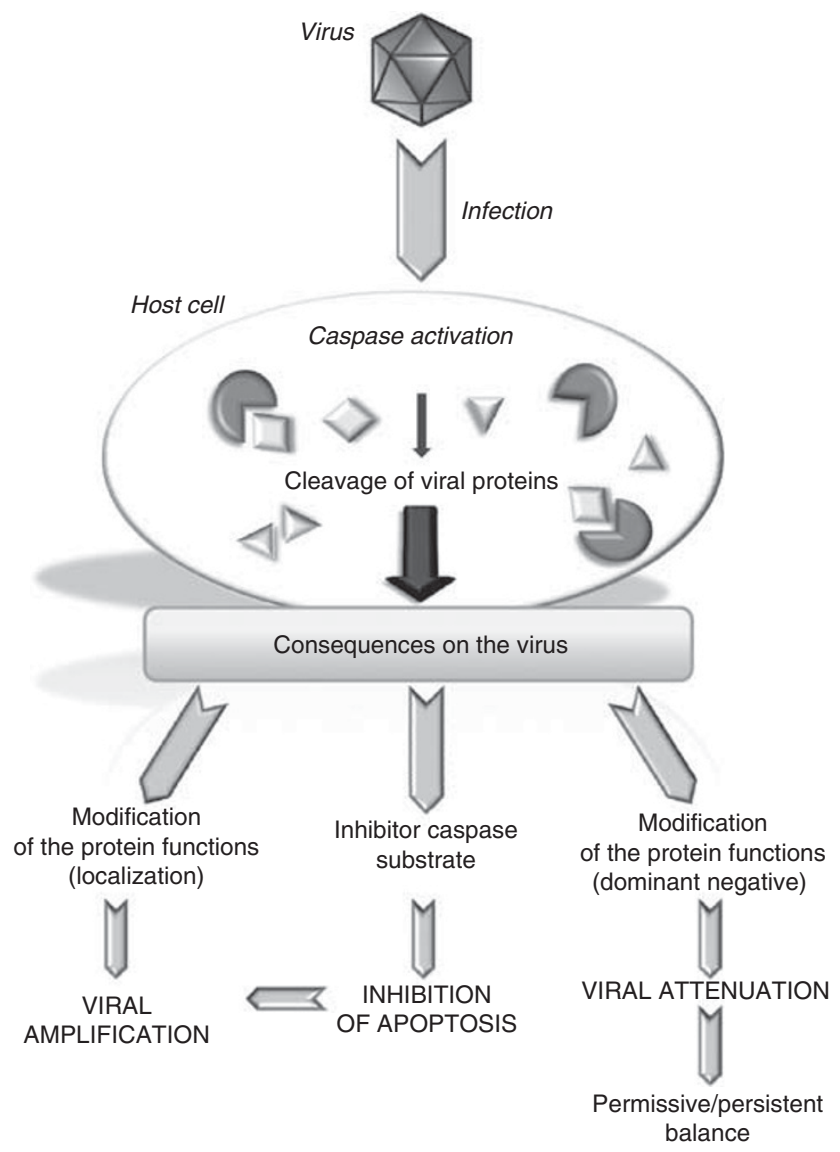

Figure 1 Functional consequences of caspase cleavages of VPs. This figure summarizes the conclusions we made about the biological relevance of caspase cleavages of VPs. A virus infecting a host cell is represented, leading to caspase activation and cleavage of one or several VPs. The consequences of such caspase cleavages on the viral life cycle are indicated, as well as the possible links between them

entities (influenza, HCV), the multitude of strains and genotypes (influenza, HCV, adenovirus) or the lack of appropriate tools such as cellular or animal models allowing to recapitulate the viral life cycle (TGEV, MCV). When caspase cleavages of viral proteins work in too mysterious ways, it is sometimes concluded that they constitute a mechanism of degradation resulting from the host fighting back the infection. However, the caspase-related products detected are mostly stable, suggesting they could be somehow involved in the viral life cycle as mentioned right above. Besides, knowing how high the mutation rates of viruses can be ${ }^{50}$ or that some viral oncoproteins (like v-Rel) adapted to resist caspase cleavage through evolution unlike their cellular counterparts, ${ }^{51}$ keeping caspase cleavage sites seems very unlikely if viruses do not somehow profit from them. As caspase cleavage of viral proteins occurs in all sorts of proteins (structural or non-structural) and in all sorts of viruses (DNA as well as RNA), this suggests that it constitutes a strong strategy to handle apoptosis. By managing to use the molecular effectors of apoptosis to protect themselves from eradication without any additional genetic information required, viruses prove once again how fascinating their adaptability can be. 


\section{Conflict of Interest}

The authors declare no conflict of interest.

Acknowledgements. This work was supported by the French institutions CNRS, Institut Pasteur de Lille and INSERM, and by grants from 'Conseil Régional Nord-Pas de Calais' and 'Ligue contre le Cancer, comité Nord'. AR was supported by a fellowship from 'Association pour la Recherche sur le Cancer'. We are grateful to Dr. Gauthier Goormachtigh for his careful reading and the helpful comments he made about this paper.

1. Barber GN. Host defense, viruses and apoptosis. Cell Death Differ 2001; 8: 113-126.

2. Danthi P. Enter the kill zone: initiation of death signaling during virus entry. Virology 2011 ; 411: 316-324.

3. Labbe K, Saleh M. Cell death in the host response to infection. Cell Death Differ 2008; 15 1339-1349.

4. Green DR, Ferguson T, Zitvogel L, Kroemer G. Immunogenic and tolerogenic cell death. Nat Rev Immunol 2009; 9: 353-363.

5. Kepp O, Senovilla L, Galluzzi L, Panaretakis T, Tesniere A, Schlemmer F et al. Viral subversion of immunogenic cell death. Cell Cycle 2009; 8: 860-869.

6. Galluzzi L, Brenner C, Morselli E, Touat Z, Kroemer G. Viral control of mitochondrial apoptosis. PLOS Pathog 2008; 4: e1000018.

7. Hay S, Kannourakis G. A time to kill: viral manipulation of the cell death program. J Gen Virol 2002; 83: 1547-1564.

8. Kaminskyy V, Zhivotovsky B. To kill or be killed: how viruses interact with the cell death machinery. J Intern Med 2010; 267: 473-482.

9. Li J, Yuan J. Caspases in apoptosis and beyond. Oncogene 2008; 27: 6194-6206.

10. Pop C, Salvesen GS. Human caspases: activation, specificity, and regulation. J Biol Chem 2009; 284: 21777-21781.

11. Best SM, Bloom ME. Caspase activation during virus infection: more than just the kiss of death? Virology 2004; 320: 191-194.

12. Clem RJ, Fechheimer M, Miller LK. Prevention of apoptosis by a baculovirus gene during infection of insect cells. Science 1991; 254: 1388-1390.

13. Bertin J, Mendrysa SM, LaCount DJ, Gaur S, Krebs JF, Armstrong RC et al. Apoptotic suppression by baculovirus P35 involves cleavage by and inhibition of a virus-induced CED-3/ICE-like protease. J Virol 1996; 70: 6251-6259.

14. Bump NJ, Hackett M, Hugunin M, Seshagiri S, Brady K, Chen P et al. Inhibition of ICE family proteases by baculovirus antiapoptotic protein p35. Science 1995; 269: 1885-1888.

15. Zhou Q, Krebs JF, Snipas SJ, Price A, Alnemri ES, Tomaselli KJ et al. Interaction of the baculovirus anti-apoptotic protein p35 with caspases. Specificity, kinetics, and characterization of the caspase/p35 complex. Biochemistry 1998; 37: 10757-10765.

16. Fisher AJ, Cruz W, Zoog SJ, Schneider CL, Friesen PD. Crystal structure of baculovirus P35: role of a novel reactive site loop in apoptotic caspase inhibition. EMBO J 1999; 18: 2031-2039.

17. Xu G, Cirilli M, Huang Y, Rich RL, Myszka DG, Wu H. Covalent inhibition revealed by the crystal structure of the caspase-8/p35 complex. Nature 2001; 410: 494-497.

18. Zoog SJ, Schiller JJ, Wetter JA, Chejanovsky N, Friesen PD. Baculovirus apoptotic suppressor $\mathrm{P} 49$ is a substrate inhibitor of initiator caspases resistant to $\mathrm{P} 35$ in vivo. EMBO J 2002; 21: 5130-5140.

19. Guy MP, Friesen PD. Reactive-site cleavage residues confer target specificity to baculovirus P49, a dimeric member of the P35 family of caspase inhibitors. J Virol 2008; 82 7504-7514.

20. Yan F, Xia D, Lv S, Qi Y, Xu H. Functional analysis of the orf390 gene of the white spot syndrome virus. Virus Res 2010; 151: 39-44.

21. Leu JH, Chen LL, Lin YR, Kou GH, Lo CF. Molecular mechanism of the interactions between white spot syndrome virus anti-apoptosis protein AAP-1 (WSSV449) and shrimp effector caspase. Dev Comp Immunol 2010; 34: 1068-1074.

22. Best SM, Wolfinbarger JB, Bloom ME. Caspase activation is required for permissive replication of Aleutian mink disease parvovirus in vitro. Virology 2002; 292: 224-234.

23. Best SM, Shelton JF, Pompey JM, Wolfinbarger JB, Bloom ME. Caspase cleavage of the nonstructural protein NS1 mediates replication of Aleutian mink disease parvovirus. J Virol 2003; 77: 5305-5312.

24. Moody CA, Laimins LA. Human papillomaviruses activate the ATM DNA damage pathway for viral genome amplification upon differentiation. PLoS Pathog 2009; 5: e1000605.

25. Moody CA, Fradet-Turcotte A, Archambault J, Laimins LA. Human papillomaviruses activate caspases upon epithelial differentiation to induce viral genome amplification. Proc Natl Acad Sci USA 2007; 104: 19541-19546.

26. Fei JW, de Villiers EM. Degradation of HPV20E6 by p53: delta Np63 alpha and mutant p53R248W protect the wild type p53 mediated caspase-degradation. Int J Cancer 2008; 123: $108-116$.
27. Banos-Lara Mdel R, Mendez E. Role of individual caspases induced by astrovirus on the processing of its structural protein and its release from the cell through a non-lytic mechanism. Virology 2010; 401: 322-332.

28. Mendez E, Salas-Ocampo E, Arias CF. Caspases mediate processing of the capsid precursor and cell release of human astroviruses. J Virol 2004; 78: 8601-8608.

29. Diemer C, Schneider M, Seebach J, Quaas J, Frosner G, Schatzl HM et al. Cell typespecific cleavage of nucleocapsid protein by effector caspases during SARS coronavirus infection. J Mol Biol 2008; 376: 23-34.

30. Majerciak V, Kruhlak M, Dagur PK, McCoy Jr JP, Zheng ZM. Caspase-7 cleavage of Kaposi sarcoma-associated herpesvirus ORF57 confers a cellular function against viral lytic gene expression. J Biol Chem 2010; 285: 11297-11307.

31. Cheng F, Chen AY, Best SM, Bloom ME, Pintel D, Qiu J. The capsid proteins of Aleutian mink disease virus activate caspases and are specifically cleaved during infection. $J$ Virol 2010; 84: 2687-2696.

32. Karlberg H, Tan YJ, Mirazimi A. Induction of caspase activation and cleavage of the viral nucleocapsid protein in different cell types during Crimean-Congo hemorrhagic fever virus infection. J Biol Chem 2011; 286: 3227-3234.

33. Zhirnov O, Bukrinskaya AG. Nucleoproteins of animal influenza viruses, in contrast to those of human strains, are not cleaved in infected cells. J Gen Virol 1984; 65 (Part 6): 1127-1134.

34. Zhirnov OP, Konakova TE, Garten W, Klenk H. Caspase-dependent N-terminal cleavage of influenza virus nucleocapsid protein in infected cells. J Virol 1999; 73: 10158-10163.

35. Lipatov AS, Yen HL, Salomon R, Ozaki H, Hoffmann E, Webster RG. The role of the $\mathrm{N}$-terminal caspase cleavage site in the nucleoprotein of influenza $A$ virus in vitro and in vivo. Arch Virol 2008; 153: 427-434.

36. Zhirnov OP, Konakova TE, Wolff T, Klenk HD. NS1 protein of influenza A virus downregulates apoptosis. J Virol 2002; 76: 1617-1625.

37. Zhirnov OP, Syrtzev VV. Influenza virus pathogenicity is determined by caspase cleavage motifs located in the viral proteins. J Mol Genet Med 2009; 3: 124-132.

38. Zhirnov OP, Klenk HD. Alterations in caspase cleavage motifs of NP and M2 proteins attenuate virulence of a highly pathogenic avian influenza virus. Virology 2009; 394: 57-63.

39. Goh PY, Tan YJ, Lim SP, Lim SG, Tan YH, Hong WJ. The hepatitis C virus core protein interacts with NS5A and activates its caspase-mediated proteolytic cleavage. Virology 2001; 290: 224-236.

40. Kalamvoki M, Georgopoulou U, Mavromara P. The NS5A protein of the hepatitis C virus genotype $1 \mathrm{a}$ is cleaved by caspases to produce $\mathrm{C}$-terminal-truncated forms of the protein that reside mainly in the cytosol. J Biol Chem 2006; 281: 13449-13462.

41. Kalamvoki M, Mavromara P. Calcium-dependent calpain proteases are implicated in processing of the hepatitis C virus NS5A protein. J Virol 2004; 78: 11865-11878.

42. Satoh S, Hirota M, Noguchi T, Hijikata M, Handa H, Shimotohno K. Cleavage of hepatitis C virus nonstructural protein $5 \mathrm{~A}$ by a caspase-like protease(s) in mammalian cells. Virology 2000; 270: 476-487.

43. Eleouet JF, Chilmonczyk S, Besnardeau L, Laude H. Transmissible gastroenteritis coronavirus induces programmed cell death in infected cells through a caspase-dependent pathway. J Virol 1998; 72: 4918-4924.

44. Eleouet JF, Slee EA, Saurini F, Castagne N, Poncet D, Garrido C et al. The viral nucleocapsid protein of transmissible gastroenteritis coronavirus (TGEV) is cleaved by caspase-6 and -7 during TGEV-induced apoptosis. J Virol 2000; 74: 3975-3983.

45. Al-Molawi N, Beardmore VA, Carter MJ, Kass GE, Roberts LO. Caspase-mediated cleavage of the feline calicivirus capsid protein. J Gen Virol 2003; 84: 1237-1244.

46. Roberts LO, Al-Molawi N, Carter MJ, Kass GE. Apoptosis in cultured cells infected with feline calicivirus. Ann NY Acad Sci 2003; 1010: 587-590.

47. Grand RJ, Schmeiser K, Gordon EM, Zhang X, Gallimore PH, Turnell AS. Caspasemediated cleavage of adenovirus early region 1 A proteins. Virology 2002; 301: 255-271.

48. Shisler JL, Moss B. Molluscum contagiosum virus inhibitors of apoptosis: the MC159 V-FLIP protein blocks Fas-induced activation of procaspases and degradation of the related MC160 protein. Virology 2001; 282: 14-25.

49. Munger J, Hagglund R, Roizman B. Infected cell protein no. 22 is subject to proteolytic cleavage by caspases activated by a mutant that induces apoptosis. Virology 2003; 305 : 364-370.

50. Barkett M, Dooher JE, Lemonnier L, Simmons L, Scarpati JN, Wang Y et al. Three mutations in v-Rel render it resistant to cleavage by cell-death protease caspase-3. Biochim Biophys Acta 2001; 1526: 25-36.

51. Sanjuan R, Nebot MR, Chirico N, Mansky LM, Belshaw R. Viral mutation rates. J Virol 2010; 84: 9733-9748.

52. Wang $\mathrm{L}$, Zhi B, Wu W, Zhang X. Requirement for shrimp caspase in apoptosis against virus infection. Dev Comp Immunol 2008; 32: 706-715.

Cell Death and Disease is an open-access journal published by Nature Publishing Group. This work is licensed under the Creative Commons Attribution-Noncommercial-No Derivative Works 3.0 Unported License. To view a copy of this license, visit http://creativecommons.org/licenses/by-nc-nd/3.0/ 